\title{
How does mindfulness training improve moral cognition: A theoretical and experimental framework for the study of embodied ethics
}

Mindfulness meditation has been primarily studied within the context of individual's psychological well being and/or in relation to it's potential to enhance cognitive skills such as attention and working memory[1]. However, in Buddhism, mindfulness is used as a tool to cultivate wholesome actions, and as a means to promote virtuous, prosocial qualities[2]. In this article, we postulate that heightened awareness of physiological and mental phenomena following mindfulness training may contribute to altered processing of morally relevant information and promote moral action. We will first briefly summarize neuroscientific investigations into moral cognition, and provide a theoretical and an experimental framework for the investigation of the relationship between mindfulness and ethical behavior.

\section{Neuroscientific Investigations Into Moral Cognition}

Morality and the moral nature of human beings have been in the center of philosophical discussions for millennia. In recent years, the complex psychological and neurobiological mechanisms underlying human morality have also been a quest for psychology and modern neuroscience. Rooted in the cognitive-developmental approach of Piaget and Kohlberg, morality was initially regarded as a traitlike ability that depend upon cognitive process[3]. In psychology, the main line of research focused on explicit moral reasoning and deliberative decision-making. More recently, however, the role of implicit processes and/or moral emotions has captured attention[4,5]. Consequently, the discipline has witnessed a burgeoning of models that amalgamate affective and cognitive components into a unified model for moral action[6-8]

From a neurocognitive perspective, morality relies on several domain general processes including a control of impulses and urges, representation of mental states, imagining the future, and other higher level cognitive processes such as reasoning and decision making $[9,10]$. It has been examined using a variety of experimental paradigms ranging from tasks that focus on moral reasoning and decision-making using highly evocative moral dilemmas (e.g. would you sacrifice one person to save the life of five others)[11], to tasks that isolate perceptual and attentional influences on moral processing in the absence of decision making (e.g. tasks that involve passive viewing of immoral actions[12,13] or implicit associations[14]). The influence of moral intuitions (concern for harm, fairness, purity, authority, and in-group favoritism) have also been evaluated experimentally [15], as well as prosocial behaviors such as cooperation and altruism using economic exchange games[16].

The initial investigations into the neural correlates of morality used functional brain imaging techniques while participants made explicit decisions about highly evocative moral dilemmas. These researchers discovered that moral judgments were guided by the interplay of fast automatic processes (affective/intuitive) and slow controlled processes (reasoning)[11]. Neuroimaging studies conducted with both healthy controls and patients with morally aberrant behavior have further contributed to the elucidation of these automatic processes in guiding our moral actions[17-19]. Corroborating the role of affect, patients with lesions of ventromedial prefrontal cortex - a region critical for emotional response 
and emotion regulation- displayed an abnormally 'utilitarian' pattern of judgments on moral dilemmas (i.e. were more likely to sacrifice one person's life to save multiple other lives) [20,21] and patients with antisocial behaviors displayed impairments in affective component of moral processing, without any impairments in their reasoning ability[22].

Although the role of emotions in guiding moral behavior is now established, it is still unclear how the affective and cognitive components interact to influence moral action. While some researchers suggest a conflict between responses driven by affective and cognitive processes[11], whereby each process favors a certain outcome (e.g. deontological vs. utilitarian inclinations), others argue that these judgments are predominantly driven by automatic, intuitive process, with deliberative reasoning only providing a post hoc justification of the decision[15]. While the debate on the complex interaction between affective and cognitive processes still continues, multiple dynamic models that attempt to capture the variety of situations that reflect real life moral encounters have also been introduced[10,23,24].

Moving beyond the dichotomy of affect versus reason, these dynamic models have incorporated contextual modulations. They differentiated situations that require quick affect laden reactions (e.g. witnessing a situation that demand an immediate response) from situations which allow for decisions based on deliberative reasoning (e.g. responding to a moral dilemma scenario) [25] and underline the flexible use of many different component processes including basic perception[26]. These models have largely been supported by research investigating perceptual[27,28] and attentional[29,30] influences to moral behaviors. Providing support to this dynamic view of moral action, a recent meta-analysis of functional neuroimaging studies that compared neural activity associated with the passive viewing of morally laden stimuli to neural activity during moral decision making [31] demonstrated that tasks involving passive viewing of moral stimuli were associated with activation of emotional and visual association cortices, including the amygdala and ventral temporal brain regions. In contrast, tasks requiring active moral judgments and mental simulations to attribute mental states to others, engaged regions of the default network. These dissociable patterns of brain activity underlined the significance of specific contextual dynamics of moral processing, supporting the dynamic view of moral cognition.

Relying on the view that domain-general, large-scale intrinsic brain networks underlie the neural substrates of emotional, social, and cognitive phenomena[32], Sevinc and colleagues further expanded the model by predicting that the salience/ventral attention network would be associated with the initial detection of moral content, while the subsequent modulation of downstream brain regions would be associated with the processing of morally relevant information[33]. In this model, the initial detection is presumed to be critical for moral action, especially in initiating a more context-specific and goal-directed processing of the moral content, marked by the engagement of default and fronto-parietal executive control networks[34-36]. In an fMRI investigation, they found evidence for this proposition and demonstrated that morally relevant information is detected automatically and tagged by regions of the salience network. In line with it's role in dynamic switching between internally and externally directed cognition[36], the salience network involvement was succeeded by the engagement of default and frontoparietal control networks, potentially reflecting associative, integrative processes required for further processing of morally-relevant information. 
Here we utilize this dynamic view of moral cognition to build a theoretical framework for the investigation of the relationship between mindfulness training and moral action. We propose that the heightened awareness of physiological and mental phenomena, attained through mindfulness meditation, may contribute to alterations in the processing of morally relevant information and promote moral action.

\section{A neuroscientific framework for the investigation embodied ethics}

Mindfulness meditation has primarily been studied within the context of psychological outcomes and/or in relation to it's potential to enhance cognitive abilities[37,38]. Several researchers and Buddhist scholars, however, have underlined the joint nature of mindfulness and ethics; and mindfulness meditation's potential role in enhancing wholesome behaviors [39,40]. In line with these assertions, investigations into the relationship between mindfulness and ethics have successfully demonstrated mindfulness training dependent improvements in moral reasoning ability[41,42], prosociality[43,44], as well as an association between trait levels of mindfulness and ethical decision making[45]. Improvements in prosociality have also been documented following other contemplative tradition based interventions such as compassion training[46,47].

Given the range of cognitive components that constitute moral cognition and the multiplicity of proposed mechanisms of action associated with mindfulness meditation[48,49], it is reasonable to hypothesize that multiple mechanisms including enhanced attentional capacity, interoceptive awareness, emotion regulation, and a change in perspective of the self synergistically contribute to enhanced moral action and prosocial behavior. Here we postulate that the dynamic model of moral cognition together with the distributed brain network perspective of mindfulness may provide a framework for the investigation of these multiple mechanisms through which mindfulness impacts ethical behaviors.

This distributed brain network perspective has already been employed to investigate neural correlates of mindfulness meditation. Researchers have successfully demonstrated default network engagement during mind wandering periods; the salience network engagement during the awareness of the mind-wandering, and the frontoparietal executive network engagement when disengaging from mind wandering and redirecting attention, with dorsolateral prefrontal cortex activity persisting into focused attention meditation[50,51]. Underlying long-term changes following mindfulness training, the repeated engagement of these networks may lead to lasting adaptations within these networks and may modulate cognition via alterations in network functioning during non-meditative periods. In line with this proposition, long-term meditators exhibit increased resting state connectivity within the default network[52,53] as well as in attentional networks, and between the attentional networks and medial frontal regions[54,55]. Our model hinges on these findings and further posits that functional changes in these distributed brain networks may mediate alterations in moral processing, leading to ethical action[56].

A crucial aspect of moral behavior is the initial detection of the moral-relevant situation (e.g. a person in pain). For social animals, such situations are by definition salient events that require regulation of behavior as well as one's homeostatic state. Mindfulness training may influence moral processing by enhancing available resources for this initial step, i.e. the detection of morally-relevant stimuli, mediated by enhanced activity in the salience network[57]. Central structures of this network include dorsal 
anterior cingulate and orbital frontoinsula, which have been associated with interoceptive, autonomic processing, while its limbic nodes including amygdala are associated with emotion, homeostatic regulation, and reward[57].These regions have repeatedly been implicated in mindfulness meditation[58-63]. Mindfulness training has been shown to augment contribution of interoception to perceptual experience as well[64]. In line with these findings and embodied nature of ethical action[56], here, we propose that an enhanced awareness of and attention to morally-relevant internal and external cues, marked by enhanced salience network engagement, together with changes in its modulatory inputs to default and/or frontoparietal control networks may provide an excellent framework to investigate mechanisms that underlie changes in moral behavior following mindfulness training and for the study of embodied ethics.

According to our model, the initial detection of moral content initiates a context-specific and goaldirected processing. These processes include self-related and regulatory processes, marked by the engagement of the default and frontoparietal control networks. The default network, especially its core regions, has been implicated in self-referential processing [65], and default network activity coupled with the frontoparietal control network supports goal-directed cognition[35]. In line with our proposition, Weng and colleagues[46] demonstrated a relationship between activity in regions of the fronto-parietal executive control network during exposure to images depicting human suffering and altruistic behavior in an economic redistribution game. In line with our proposed model, they argued that an increased engagement of these regions following compassion training may reflect greater sustained attention and goal maintenance to help others, as well as an enhanced integration of external (such as others' suffering) and internal information (the goal to help), potentially leading to improvements in moral behavior.

It is important to distinguish between brief secular training programs that incorporate compassion or mindfulness meditation and long-term practitioners obtaining training from traditional Buddhist teachers. First, while there is some overlap between the proposed mechanisms of compassion and mindfulness meditation, each has been associated with different mechanisms of action[63]. Second, since ethics are a very prominent component of Buddhism, studies with long-term practitioners must take into account those practitioners' explicit efforts to become more ethical. Thus, a randomized controlled investigation of secular mindfulness training is essential to investigate how mindfulness in and of itself contributes to enhanced ethics. Such an investigation may validate the use of contemplative practices for catalyzing change at the interpersonal level and ultimately contribute to the development of better intervention programs to enhance moral and prosocial behaviors.

\section{References}

[1] J. Kabat-Zinn, Mindfulness-based interventions in context: Past, present, and future, Clinical Psychology: Science \& Practice. 10 (2003) 144-156.

[2] B. Bodhi, What does mindfulness really mean? A canonical perspective, Contemporary Buddhism. 12 (2011) 19-39. doi:10.1080/14639947.2011.564813.

- The author provides an account of mindfulness in the Buddhist tradition and underlines its ethical aspects, mentioning that it is more than 'bare attention'. 
[3] L. Kohlberg, R.H. Hersh, Moral development: A review of the theory, Theory Into Practice. 16 (1977) 53-59. doi:10.1080/00405847709542675.

[4] J. Moll, R. De Oliveira-Souza, R. Zahn, The neural basis of moral cognition: sentiments, concepts, and values, Ann. N. Y. Acad. Sci. 1124 (2008) 161-180. doi:10.1196/annals.1440.005.

[5] J. Moll, R. de Oliveira-Souza, P.J. Eslinger, I.E. Bramati, J. Mourão-Miranda, P.A. Andreiuolo, L. Pessoa, The neural correlates of moral sensitivity: a functional magnetic resonance imaging investigation of basic and moral emotions, J. Neurosci. 22 (2002) 2730-2736. doi:20026214.

[6] L. Young, M. Koenigs, Investigating emotion in moral cognition: a review of evidence from functional neuroimaging and neuropsychology, Br. Med. Bull. 84 (2007) 69-79. doi:10.1093/bmb/ldm031.

[7] L. Pascual, P. Rodrigues, D. Gallardo-Pujol, How does morality work in the brain? A functional and structural perspective of moral behavior, Front Integr Neurosci. 7 (2013). doi:10.3389/fnint.2013.00065.

[8] J.W. Buckholtz, R. Marois, The roots of modern justice: cognitive and neural foundations of social norms and their enforcement, Nat. Neurosci. 15 (2012) 655-661. doi:10.1038/nn.3087.

[9] L. Young, J. Dungan, Where in the brain is morality? Everywhere and maybe nowhere, Soc Neurosci. 7 (2012) 1-10. doi:10.1080/17470919.2011.569146.

[10] J. Moll, R. Zahn, R. de Oliveira-Souza, F. Krueger, J. Grafman, Opinion: the neural basis of human moral cognition, Nat. Rev. Neurosci. 6 (2005) 799-809. doi:10.1038/nrn1768.

[11]J. Greene, J. Haidt, How (and where) does moral judgment work?, Trends Cogn. Sci. (Regul. Ed.). 6 (2002) 517-523.

[12] C.L. Harenski, O. Antonenko, M.S. Shane, K.A. Kiehl, A functional imaging investigation of moral deliberation and moral intuition, Neuroimage. 49 (2010) 2707-2716. doi:10.1016/j.neuroimage.2009.10.062.

[13] C.L. Harenski, S. Hamann, Neural correlates of regulating negative emotions related to moral violations, Neuroimage. 30 (2006) 313-324. doi:10.1016/j.neuroimage.2005.09.034.

[14] Q. Luo, M. Nakic, T. Wheatley, R. Richell, A. Martin, R.J.R. Blair, The neural basis of implicit moral attitude-An IAT study using event-related fMRI, NeuroImage. 30 (2006) 1449-1457. doi:10.1016/j.neuroimage.2005.11.005.

- $\quad$ Researchers show neural correlates associated with implicit moral attitude using a moral implicit association task, by indexing increased neural response in the right amygdala and the ventromedial orbitofrontal cortex as a function of stimulus intensity.

[15] J. Haidt, The emotional dog and its rational tail: a social intuitionist approach to moral judgment, Psychol Rev. 108 (2001) 814-834.

[16]C.T. Dawes, P.J. Loewen, D. Schreiber, A.N. Simmons, T. Flagan, R. McElreath, S.E. Bokemper, J.H. Fowler, M.P. Paulus, Neural basis of egalitarian behavior, PNAS. 109 (2012) 6479-6483. doi:10.1073/pnas.1118653109.

[17] S. Green, M.A.L. Ralph, J. Moll, E.A. Stamatakis, J. Grafman, R. Zahn, Selective functional integration between anterior temporal and distinct fronto-mesolimbic regions during guilt and indignation, Neuroimage. 52 (2010) 1720-1726. doi:10.1016/j.neuroimage.2010.05.038.

[18] J.D. Greene, S.A. Morelli, K. Lowenberg, L.E. Nystrom, J.D. Cohen, Cognitive load selectively interferes with utilitarian moral judgment, Cognition. 107 (2008) 1144-1154. doi:10.1016/j.cognition.2007.11.004.

[19] S. Berthoz, J.L. Armony, R.J.R. Blair, R.J. Dolan, An fMRI study of intentional and unintentional (embarrassing) violations of social norms, Brain. 125 (2002) 1696-1708. 
[20] E. Ciaramelli, M. Muccioli, E. Làdavas, G. di Pellegrino, Selective deficit in personal moral judgment following damage to ventromedial prefrontal cortex, Soc Cogn Affect Neurosci. 2 (2007) 84-92. doi:10.1093/scan/nsm001.

[21] M. Koenigs, L. Young, R. Adolphs, D. Tranel, F. Cushman, M. Hauser, A. Damasio, Damage to the prefrontal cortex increases utilitarian moral judgements, Nature. 446 (2007) 908-911. doi:10.1038/nature05631.

[22] A. Raine, Y. Yang, Neural foundations to moral reasoning and antisocial behavior, Soc Cogn Affect Neurosci. 1 (2006) 203-213. doi:10.1093/scan/nsl033.

[23] C.E. Forbes, J. Grafman, The Role of the Human Prefrontal Cortex in Social Cognition and Moral Judgment, Annual Review of Neuroscience. 33 (2010) 299-324. doi:10.1146/annurev-neuro060909-153230.

[24] J.J. Van Bavel, O. FeldmanHall, P. Mende-Siedlecki, The neuroscience of moral cognition: from dual processes to dynamic systems, Current Opinion in Psychology. 6 (2015) 167-172. doi:10.1016/j.copsyc.2015.08.009.

- In this article, the authors make a distinction between real versus hypothetical moral situations and demonstrate how social context modulates the dynamic processes underlying real moral judgments . They also advocate a dynamic view of moral judgment, beginning from perception.

[25] B. Monin, D.A. Pizarro, J.S. Beer, Deciding versus reacting: Conceptions of moral judgment and the reason-affect debate., Review of General Psychology. 11 (2007) 99-111. doi:10.1037/1089-2680.11.2.99.

[26] A.P. Gantman, J.J. Van Bavel, The moral pop-out effect: Enhanced perceptual awareness of morally relevant stimuli, Cognition. 132 (2014) 22-29. doi:10.1016/j.cognition.2014.02.007.

[27] A.P. Gantman, J.J. Van Bavel, Moral perception, Trends in Cognitive Sciences. 19 (2015) 631633.

[28] A.P. Gantman, J.J. Van Bavel, Exposure to justice diminishes moral perception, Journal of Experimental Psychology: General. 145 (2016) 1728-1739. doi:10.1037/xge0000241.

[29] F. van Nunspeet, N. Ellemers, B. Derks, S. Nieuwenhuis, Moral concerns increase attention and response monitoring during IAT performance: ERP evidence, Soc Cogn Affect Neurosci. 9 (2014) 141-149. doi:10.1093/scan/nss118.

[30] L.F. Van Dillen, R.C. van der Wal, K. van den Bos, On the Role of Attention and Emotion in Morality: Attentional Control Modulates Unrelated Disgust in Moral Judgments, Pers Soc Psychol Bull. 38 (2012) 1222-1231. doi:10.1177/0146167212448485.

[31] G. Sevinc, R.N. Spreng, Contextual and Perceptual Brain Processes Underlying Moral Cognition: A Quantitative Meta-Analysis of Moral Reasoning and Moral Emotions, PLOS ONE. 9 (2014) e87427. doi:10.1371/journal.pone.0087427.

[32] L.F. Barrett, A. Satpute, Large-scale brain networks in affective and social neuroscience: Towards an integrative functional architecture of the brain, Curr Opin Neurobiol. 23 (2013) 361-372. doi:10.1016/j.conb.2012.12.012.

[33] G. Sevinc, H. Gurvit, R.N. Spreng, Salience network engagement with the detection of morally laden information, Social Cognitive and Affective Neuroscience. (2017). https://academic.oup.com/scan/article/3064491 (accessed May 31, 2017).

[34] V. Menon, L.Q. Uddin, Saliency, switching, attention and control: a network model of insula function, Brain Struct Funct. 214 (2010) 655-667. doi:10.1007/s00429-010-0262-0. 
[35] R.N. Spreng, W.D. Stevens, J.P. Chamberlain, A.W. Gilmore, D.L. Schacter, Default network activity, coupled with the frontoparietal control network, supports goal-directed cognition, Neuroimage. 53 (2010) 303-317. doi:10.1016/j.neuroimage.2010.06.016.

[36] L.Q. Uddin, Salience processing and insular cortical function and dysfunction, Nat. Rev. Neurosci. 16 (2015) 55-61. doi:10.1038/nrn3857.

[37] R.A. Baer, Mindfulness training as a clinical intervention: A conceptual and empirical review, Clinical Psychology: Science \& Practice. 10 (2003) 125-143.

[38] P. Grossman, L. Niemann, S. Schmidt, H. Walach, Mindfulness-based stress reduction and health benefits. A meta-analysis, Journal of Psychosomatic Research. 57 (2004) 35-43.

[39] M.T. Greenberg, J.L. Mitra, From Mindfulness to Right Mindfulness: the Intersection of Awareness and Ethics, Mindfulness. 6 (2015) 74-78. doi:10.1007/s12671-014-0384-1.

[40] B. Bodhi, What does mindfulness really mean? A canonical perspective, Contemporary Buddhism. 12 (2011) 19-39. doi:10.1080/14639947.2011.564813.

[41] S.L. Shapiro, H. Jazaieri, P.R. Goldin, Mindfulness-based stress reduction effects on moral reasoning and decision making, The Journal of Positive Psychology. 7 (2012) 504-515. doi:10.1080/17439760.2012.723732.

- $\quad$ Researchers provide an account of mechanisms through which mindfulness meditation improve decision-making, emphasizing the ability to realize that a situation has a moral component awareness, change in perspective of the self such as a less egocentric frame of reference. They provide preliminary evidence that MBSR results in improvements in moral reasoning and ethical decision making in 2-month follow-up.

[42] A. Pandey, R. Chandwani, A. Navare, How can mindfulness enhance moral reasoning? An examination using business school students, Business Ethics: A European Review. 27 (2018) 56-71. doi:10.1111/beer.12171.

[43] P. Condon, G. Desbordes, W.B. Miller, D. DeSteno, Meditation increases compassionate responses to suffering, Psychol Sci. 24 (2013) 2125-2127. doi:10.1177/0956797613485603.

- In this article, researchers demonstrated that participating a brief meditation training increase the likelihood of compassionate response (increase in the number of participants who offered their seats to relieve a person's suffering) as opposed to a wait-list control. They also examined whether participants response differed depending on whether they practiced mindfulness meditation or compassion meditation and demonstrated that there were no difference between the two interventions.

[44] J.N. Donald, B.K. Sahdra, B. Van Zanden, J.J. Duineveld, P.W.B. Atkins, S.L. Marshall, J. Ciarrochi, Does your mindfulness benefit others? A systematic review and meta-analysis of the link between mindfulness and prosocial behaviour, Br J Psychol. (2018). doi:10.1111/bjop.12338.

[45] N.E. Ruedy, M.E. Schweitzer, In the Moment: The Effect of Mindfulness on Ethical Decision Making, J Bus Ethics. 95 (2010) 73-87. doi:10.1007/s10551-011-0796-y.

[46] H.Y. Weng, A.S. Fox, A.J. Shackman, D.E. Stodola, J.Z.K. Caldwell, M.C. Olson, G.M. Rogers, R.J. Davidson, Compassion training alters altruism and neural responses to suffering, Psychol Sci. 24 (2013) 1171-1180. doi:10.1177/0956797612469537.

[47] S. Leiberg (Di Pietrantonio, O. Klimecki, T. Singer, Short-Term Compassion Training Increases Prosocial Behavior in a Newly Developed Prosocial Game, PloS One. 6 (2011) e17798. doi:10.1371/journal.pone.0017798.

[48] B.K. Hölzel, S.W. Lazar, T. Gard, Z. Schuman-Olivier, D.R. Vago, U. Ott, How Does Mindfulness Meditation Work? Proposing Mechanisms of Action From a Conceptual and Neural Perspective, Perspectives on Psychological Science. 6 (2011) 537-559. doi:10.1177/1745691611419671. 
- Researchers give an account of mechanisms through which mindfulness meditation can exert its salubrious effects. These include (a) attention regulation, (b) body awareness, (c) emotion regulation (including reappraisal and exposure, extinction, and reconsolidation), and (d) change in perspective on the self.

[49] D.R. Vago, D.A. Silbersweig, Self-awareness, self-regulation, and self-transcendence (S-ART): a framework for understanding the neurobiological mechanisms of mindfulness, Front Hum Neurosci. 6 (2012). doi:10.3389/fnhum.2012.00296.

[50] W. Hasenkamp, C.D. Wilson-Mendenhall, E. Duncan, L.W. Barsalou, Mind wandering and attention during focused meditation: A fine-grained temporal analysis of fluctuating cognitive states, NeuroImage. 59 (2012) 750-760. doi:10.1016/j.neuroimage.2011.07.008.

[51] M. Ellamil, K.C.R. Fox, M.L. Dixon, S. Pritchard, R.M. Todd, E. Thompson, K. Christoff, Dynamics of neural recruitment surrounding the spontaneous arising of thoughts in experienced mindfulness practitioners, Neuroimage. 136 (2016) 186-196. doi:10.1016/j.neuroimage.2016.04.034.

[52] K.A. Garrison, T.A. Zeffiro, D. Scheinost, R.T. Constable, J.A. Brewer, Meditation leads to reduced default mode network activity beyond an active task, Cogn Affect Behav Neurosci. 15 (2015) 712-720. doi:10.3758/s13415-015-0358-3.

[53] V.A. Taylor, V. Daneault, J. Grant, G. Scavone, E. Breton, S. Roffe-Vidal, J. Courtemanche, A.S. Lavarenne, G. Marrelec, H. Benali, M. Beauregard, Impact of meditation training on the default mode network during a restful state, Soc Cogn Affect Neurosci. 8 (2013) 4-14. doi:10.1093/scan/nsr087.

[54] W. Hasenkamp, L.W. Barsalou, Effects of Meditation Experience on Functional Connectivity of Distributed Brain Networks, Front Hum Neurosci. 6 (2012). doi:10.3389/fnhum.2012.00038.

[55] A. Doll, B.K. Hölzel, C.C. Boucard, A.M. Wohlschläger, C. Sorg, Mindfulness is associated with intrinsic functional connectivity between default mode and salience networks, Front Hum Neurosci. 9 (2015). doi:10.3389/fnhum.2015.00461.

[56] F.J. Varela, Ethical know-how: Action, wisdom, and cognition, Stanford University Press, 1999.

[57]W.W. Seeley, V. Menon, A.F. Schatzberg, J. Keller, G.H. Glover, H. Kenna, A.L. Reiss, M.D. Greicius, Dissociable intrinsic connectivity networks for salience processing and executive control, J. Neurosci. 27 (2007) 2349-2356. doi:10.1523/JNEUROSCI.5587-06.2007.

[58] S.W. Lazar, G. Bush, R.L. Gollub, G.L. Fricchione, G. Khalsa, H. Benson, Functional brain mapping of the relaxation response and meditation, Neuroreport. 11 (2000) 1581-5.

[59] S.W. Lazar, C.E. Kerr, R.H. Wasserman, J.R. Gray, D.N. Greve, M.T. Treadway, M. McGarvey, B.T. Quinn, J.A. Dusek, H. Benson, S.L. Rauch, C.I. Moore, B. Fischl, Meditation experience is associated with increased cortical thickness, Neuroreport. 16 (2005) 1893-7.

[60] B.K. Holzel, J. Carmody, K.C. Evans, E.A. Hoge, J.A. Dusek, L. Morgan, R.K. Pitman, S.W. Lazar, Stress reduction correlates with structural changes in the amygdala, Soc Cogn Affect Neurosci. 5 (2010) 11-7. doi:nsp034 [pii] 10.1093/scan/nsp034.

[61] B.K. Holzel, J. Carmody, M. Vangel, C. Congleton, S.M. Yerramsetti, T. Gard, S.W. Lazar, Mindfulness practice leads to increases in regional brain gray matter density, Psychiatry Res. 191 (2011) 36-43. doi:S0925-4927(10)00288-X [pii] 10.1016/j.pscychresns.2010.08.006.

[62] K.C.R. Fox, S. Nijeboer, M.L. Dixon, J.L. Floman, M. Ellamil, S.P. Rumak, P. Sedlmeier, K. Christoff, Is meditation associated with altered brain structure? A systematic review and meta-analysis of morphometric neuroimaging in meditation practitioners, Neurosci Biobehav Rev. 43 (2014) 48-73. doi:10.1016/j.neubiorev.2014.03.016. 
[63] K.C.R. Fox, M.L. Dixon, S. Nijeboer, M. Girn, J.L. Floman, M. Lifshitz, M. Ellamil, P. Sedlmeier, K. Christoff, Functional neuroanatomy of meditation: A review and meta-analysis of 78 functional neuroimaging investigations, Neurosci Biobehav Rev. 65 (2016) 208-228. doi:10.1016/j.neubiorev.2016.03.021.

[64] N.A.S. Farb, Z.V. Segal, A.K. Anderson, Mindfulness meditation training alters cortical representations of interoceptive attention, Soc Cogn Affect Neurosci. 8 (2013) 15-26. doi:10.1093/scan/nss066.

[65] J.R. Andrews-Hanna, J. Smallwood, R.N. Spreng, The default network and self-generated thought: component processes, dynamic control, and clinical relevance, Ann N Y Acad Sci. 1316 (2014) 29-52. doi:10.1111/nyas.12360. 